\title{
TOLERANCE DESIGN OF ANALOG CIRCUITS USING A BRANCH-AND-BOUND BASED APPROACH
}

\author{
MICHAEL PEHL ${ }^{\dagger}$ and HELMUT GRAEB \\ Technische Universitaet Muenchen, Munich, Germany \\ ${ }^{\dagger}$ christian.michael.pehl@mytum.de \\ Received 13 March 2012 \\ Accepted 16 May 2012 \\ Published 18 January 2013
}

\begin{abstract}
The design of circuits which are robust against variations in operating and process conditions is crucial in today's IC industry. In the analog design flow this problem can be tackled during the sizing of a new circuit. However, hardly any methods are available which support the designer to compute such a robust design if discrete parameters should be considered in this design step. Discrete parameters arise predominantly if a layout-friendly sizing should be computed in the sense that, e.g., a manufacturing grid for the transistor lengths and widths is considered or that transistor multipliers are used to allow the layout of a transistor as multifinger or common centroid structure without applying rounding operations to the carefully computed sizing. This paper presents a new Branch-and-Bound based approach which allows the automatic computation of a robust design using classical and realistic worst case analysis. The results of the sizing of three circuits show that the new approach is highly efficient. The robustness of the results computed by the new approach is validated by Monte Carlo analyses.
\end{abstract}

Keywords: Variation; tolerance; discrete; analog; branch-and-bound.

\section{Introduction}

In analog circuit sizing, a circuit schematic - either newly designed or reused from previous applications - is given. For the schematic, the design parameter values, e.g., the transistor lengths and widths, must be computed so that the circuit meets predefined performance specifications in consideration of operating conditions and yield requirements. Such a design is referred to as tolerance design in the following. Along with robustness requirements, discrete parameters must be considered in analog sizing. Such parameters predominantly appear if layout properties should be considered, e.g., if transistor lengths and widths must lie on a manufacturing grid or if transistor multipliers should be used for scaling, to model the transistor layout as 
multifinger or common centroid structure. Also the usage of new devices like FinFETs $^{1}$ requires the consideration of discrete parameters as such devices frequently can only be scaled discretely.

The low number of deterministic ${ }^{2,3}$ and stochastic ${ }^{4,5}$ approaches which can consider discrete design parameters focuses on the design of circuits with fixed operating and process conditions. Available methods to consider robustness requirements ${ }^{6,7}$ do not incorporate discrete parameters, and rounding operations are applied in practice to discretize the result. Therefore, these methods cannot solve a discrete optimization problem $^{8}$ or a discrete analog sizing task in general. Approaches for yield optimization are available $\mathrm{e}^{6,9}$ but do not consider discrete parameters either.

In practice, state-of-the-art continuous simulation based approaches ${ }^{10}$ are frequently used in solving the analog sizing task. The continuous result is than rounded to the next discrete point although this does not solve the problem in general. ${ }^{8}$ This method is referred to as continuous sizing and rounding. In contrast, the new method $^{2}$ uses an outer Branch-and-Bound approach which adds a sequence of branching constraints to the problem and causes that a discrete point is found after a finite number of recursions. In each step of the Branch-and-Bound algorithm a continuous sizing is computed using an $\mathrm{FSQP}^{11}$ approach where all parameters can be changed subject to the restrictions given by the branching constraints. Therefore, the solution can be any discrete point.

In addition an inner Branch-and-Bound is used in the new method to predict a discrete solution in each step, which is, however, accepted only if it solves the task. This can be interpreted as advanced rounding. But in contrast to rounding, it does not restrict the solution to be a discrete neighbors of the continuous result.

A successive approximation of the worst case for each performance ${ }^{7}$ is used in the new approach to consider variations in operating and process conditions, which is based on classical and realistic worst case analysis. ${ }^{6}$ The validation of simulation results by Monte Carlo analyses shows that - in contrast to the state-of-the-art approach of continuous sizing and subsequent rounding - the new, highly efficient approach can compute a tolerance design which fulfills predefined yield requirements using typical discrete design parameters.

The article is structured as follows: A formal description of the tolerance design task is given in Sec. 2. In Sec. 3, the new approach is described which is applied to three different circuits in the experiments in Sec. 4. Section 5 concludes.

\section{Problem Definition}

\subsection{Design parameters}

The parameters which can be influenced by the designer, e.g., the lengths and widths of the transistors, are referred to as design parameters and can be chosen in a physically limited range between a lower and an upper bound $\mathbf{d}_{l}$ and $\mathbf{d}_{u}$. The design 
parameter domain $\mathbb{D}_{\text {rel }}^{N}$ can be defined as

$$
\mathbb{D}_{\text {rel }}^{N}=\left\{\mathbf{d} \mid \mathbf{d}_{l} \leq \mathbf{d} \leq \mathbf{d}_{u}\right\}
$$

if the parameter values can be selected from a continuous domain. In this work also discrete design parameters should be considered which arise, e.g., if transistor multipliers, a manufacturing grid, or the number of winding turns of an integrated inductor are used. It is assumed that each circuit property can be evaluated for design parameters in a continuous (relaxed) domain $\mathbb{D}_{\text {rel }}^{N}$. This assumption is valid for many discrete design parameters, in particular for the parameters mentioned above. Nevertheless, these parameters must be discrete after sizing, i.e., each discrete parameter $d_{i}$ must be element of a discrete set

$$
\begin{aligned}
d_{i} \in \mathbb{D}_{d, i}:= & \left(\mathbb{D}_{d, i},<\right)=\left\{d_{1}, \ldots, d_{k}, \ldots, d_{n_{i}}\right\} \\
& \times \bigvee_{k \in 1, \ldots, n_{i}-1} d_{k}<d_{k+1} .
\end{aligned}
$$

It is assumed that the $n_{i}$ elements of this set can be ordered by the relation $<$. The finally computed design parameters must be taken from the mixed continuous and discrete domain $\mathbb{D}_{\text {disc }}^{N}$ with

$$
\mathbb{D}_{\text {disc }}^{N}=\left\{\mathbf{d} \in \mathbb{D}_{\text {rel }}^{N} \mid d_{i} \in \mathbb{D}_{d, i} \text { if parameter } i \text { is discrete }\right\}
$$

\subsection{Operating and process parameters}

Operating parameters are used to describe the environment of a circuit such as temperature or supply voltage. These parameters are collected in an $N_{o}$-dimensional vector $\mathbf{o}$ and are specified for a circuit by lower and upper bounds $\mathbf{o}_{l}$ and $\mathbf{o}_{u}$ :

$$
\mathbf{o} \in \mathbb{T}_{o}=\left\{\mathbf{o} \mid \mathbf{o}_{l} \leq \mathbf{o} \leq \mathbf{o}_{u}\right\} .
$$

The specified performances of the circuit must be guaranteed for each operating point inside this tolerance domain $\mathbb{T}_{o}$.

Different from the operating parameters, process parameters are used to describe variations in oxide thickness, electron mobility, threshold voltage, etc. Such effects can be modeled by $N_{s}$ normal distributed random variables s with

$$
\operatorname{pdf}(\mathbf{s})=\frac{1}{\sqrt{2 \pi}^{N_{s}} \sqrt{\operatorname{det}(\mathbf{C})}} \cdot \exp \left(-\frac{1}{2} \beta^{2}(\mathbf{s})\right)
$$

$\mathbf{C}$ is the covariance matrix. For mean values $\mathbf{s}_{0}, \beta^{2}(\mathbf{s})$ is defined as:

$$
\beta^{2}(\mathbf{s})=\left(\mathbf{s}-\mathbf{s}_{0}\right)^{T} \cdot \mathbf{C}^{-1} \cdot\left(\mathbf{s}-\mathbf{s}_{0}\right) .
$$

Given the probability density function (pdf) and the specification $f_{U}$ of a performance $f(\mathbf{s})$ in the process parameter domain (Fig. 1) such that $f(\mathbf{s}) \leq f_{U}$ should be 


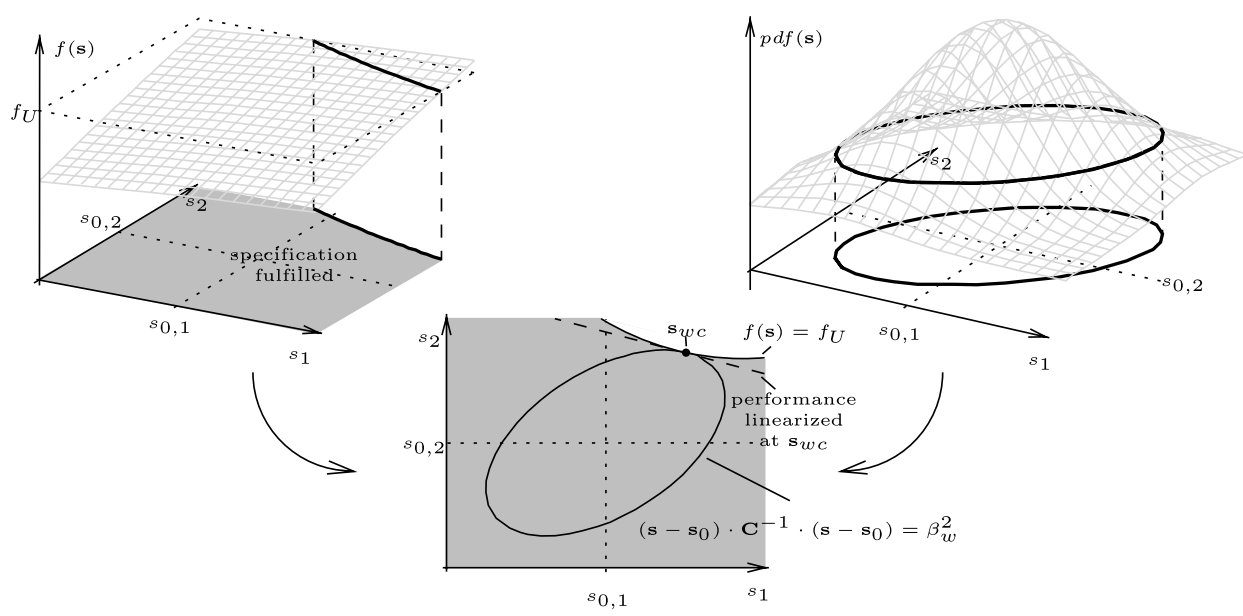

Fig. 1. $\operatorname{pdf}(\mathbf{s})$, performance $f(\mathbf{s})$, contour line for $\left(\mathbf{s}-\mathbf{s}_{0}\right) \cdot \mathbf{C}^{-1} \cdot\left(\mathbf{s}-\mathbf{s}_{0}\right)=\beta_{w}^{2}$, upper bound $f(\mathbf{s})=f_{U}$, and linearized performance at boundary point $\mathbf{s}_{\mathrm{wc}}$ of contour lines for $\operatorname{pdf}(\mathbf{s})$ and $f(\mathbf{s})$.

fulfilled, the expected parametric yield of a circuit with respect to the performance is the integral over $\operatorname{pdf}(\mathbf{s})$ in the domain where $f(\mathbf{s}) \leq f_{U}$ is met (shaded area). Using a linearization of the performance at the boundary point of the contour lines for $\operatorname{pdf}(\mathbf{s})$ and $f(\mathbf{s})=f_{U}$ (cf. Fig. 1), this yield can be approximated by the integral over this linearized area or $^{6}$

$$
Y^{\prime}=\int_{-\infty}^{\beta_{w}} \frac{1}{\sqrt{2 \pi}} \cdot \exp \left(-\frac{1}{2} \beta^{2}\right) d \beta .
$$

With (6) and (7) the yield corresponds to a certain ellipsoid defined by the value of $\beta_{w}$. E.g., $\beta_{w}=3$ corresponds to a $3 \sigma$ design, i.e., to a yield of $99.87 \%$.

For a tolerance design, the design parameters should be selected such that a certain yield is reached. With (6), (7) the required yield can be transformed into a required value for the worst case distance $\beta_{w}$ which defines a ellipsoidal contour line of (5). The yield requirement is met if the specification is fulfilled for each process parameter point in the tolerance ellipsoid $\mathbb{T}_{s}$ with

$$
\mathbb{T}_{s}=\left\{\mathbf{s} \mid\left(\mathbf{s}-\mathbf{s}_{0}\right) \cdot \mathbf{C}^{-1} \cdot\left(\mathbf{s}-\mathbf{s}_{0}\right) \leq \beta_{w}^{2}\right\} .
$$

It can be seen in Fig. 1 that — due to the linearization - an error occurs in the approximation of the yield. However, this error is negligibly small in practice.

\subsection{Tolerance design task}

In tolerance design, values of the design parameters must be assigned to fulfill all performance specifications for each operating condition and for a minimum required yield. For this purpose, the normalized demand of performance improvement $\varepsilon_{i}(\mathbf{d}, \mathbf{o}, \mathbf{s})$ 
for performance $i$ is defined which describes the amount of improvement required for a certain performance to fulfill its specification. Without loss of generality, a nonzero specification $f_{U, i}$ is assumed for each performance $f_{i}(\mathbf{d}, \mathbf{o}, \mathbf{s})$ for the remainder of this article which is an upper bound for the required performance value, i.e., $f_{i}(\mathbf{d}, \mathbf{o}, \mathbf{s})<$ $f_{U, i}$ must be fulfilled at the solution point. With this assumption, $\varepsilon_{i}(\mathbf{d}, \mathbf{o}, \mathbf{s})$ can be defined as:

$$
\varepsilon_{i}(\mathbf{d}, \mathbf{o}, \mathbf{s})=\max \left(0, \frac{f_{i}(\mathbf{d}, \mathbf{o}, \mathbf{s})-f_{U, i}}{\left|f_{U, i}\right|}\right) .
$$

I.e., $\varepsilon_{i}(\mathbf{d}, \mathbf{o}, \mathbf{s})$ is zero if the corresponding specification is met and greater than zero if not. Therefore, the tolerance design task is equivalent to the task to compute a design parameter point $\mathbf{d}^{*}$ such that for each operating parameter point in (4) and for each process parameter point in the tolerance domain defined by $\beta_{w} \varepsilon_{i}(\mathbf{d}, \mathbf{o}, \mathbf{s})=0$ is fulfilled for all $N_{f}$ performances. I.e., with (4), (8), and (9):

$$
\bigvee_{\substack{i=1, \ldots, N_{f} \\ \mathbf{o} \in \mathbb{T}_{o}, \mathbf{s} \in \mathbb{T}_{s}}} \varepsilon_{i}\left(\mathbf{d}^{*}, \mathbf{o}, \mathbf{s}\right)=0
$$

(10) is true if at $\mathbf{d}^{*}$ each performance specification is fulfilled for the corresponding worst case point $\mathbf{o}_{w, i}^{*} \in \mathbb{T}_{o}$ and $\mathbf{s}_{w, i}^{*} \in \mathbb{T}_{s}$. The worst case points for given upper bounds $f_{U, i}$ can be computed at $\mathbf{d}^{*}$ by $^{6}$

$$
\mathbf{o}_{w, i}^{*}, \mathbf{s}_{w, i}^{*}=\arg \max _{\mathbf{o} \in \mathbb{T}_{o}, \mathbf{s} \in \mathbb{T}_{s}} f_{i}\left(\mathbf{d}^{*}, \mathbf{o}, \mathbf{s}\right) .
$$

Thus, (10) is equivalent to the task to find a vector $\mathbf{d}^{*}$ such that

$$
\forall_{i=1, \ldots, N_{f}} \varepsilon_{i}\left(\mathbf{d}^{*}, \mathbf{o}_{w, i}^{*}, \mathbf{s}_{w, i}^{*}\right)=0 .
$$

To transform this multi-objective task into a scalar optimization problem, a least squares objective function $\varphi(\mathbf{d})$ is used:

$$
\varphi(\mathbf{d}):=\sum_{i=1}^{N_{f}} \varepsilon_{i}\left(\mathbf{d}, \mathbf{o}_{w, i}^{*}, \mathbf{s}_{w, i}^{*}\right)^{2} .
$$

Therefore, the tolerance design task can be reformulated as minimization problem:

$$
\min _{\mathbf{d} \in \mathbb{D}_{\text {disc }}^{N}} \varphi(\mathbf{d}) \quad \text { s.t. } \mathbf{c}(\mathbf{d}) \geq \mathbf{0} \text {. }
$$

In (14) $\mathbf{c}(\mathbf{d})$ are design rules for the circuit $^{12}$ formulated as constraints. In contrast to the performances which are evaluated at the worst case points, the constraints are considered at the nominal operating and process parameter point. The task to compute a tolerance design is solved at $\mathbf{d}^{*}$ if the objective function is zero and the 
constraints are fulfilled at this point:

$$
\varphi\left(\mathbf{d}^{*}\right)=0 \wedge \mathbf{c}\left(\mathbf{d}^{*}\right) \geq \mathbf{0} .
$$

The design at $\mathbf{d}^{*}$ fulfills the yield requirement predefined by the selection of $\beta_{w}$. To obtain higher yield, i.e., to make the circuit even more robust, $\beta_{w}$ can be increased. Better performance values can be obtained by tightening the specified values $f_{U, i}$. The accurate computation of the worst case points in (11) is computationally very expensive in practice. Therefore these points are successively linearly approximated ${ }^{7}$ in Sec. 3.1. For this purpose, the worst case operating parameter point for performance $i$ and for fixed design and process parameters $\hat{\mathbf{d}}$ and $\hat{\mathbf{s}}$ can be given:

$$
\mathbf{o}_{w, i}=\arg \max _{\mathbf{o} \in \mathbb{T}_{o}} \varepsilon_{i}(\hat{\mathbf{d}}, \mathbf{o}, \hat{\mathbf{s}}) .
$$

Accordingly, the worst case process parameter point for fixed design and operating parameters $\hat{\mathbf{d}}$ and $\hat{\mathbf{o}}$ is

$$
\mathbf{s}_{w, i}=\arg \max _{\mathbf{s} \in \mathbb{T}_{s}} \varepsilon_{i}(\hat{\mathbf{d}}, \hat{\mathbf{o}}, \mathbf{s})
$$

\section{Automatic Sizing Approach}

Two key tasks must be solved for the automatic sizing approach in this work:

(1) The discreteness of the design parameters must be considered.

(2) The worst case points must be computed efficiently.

A discrete solution for the optimization problem is computed by the Branchand-Bound approach in Sec. 3.2. Therein a tolerance design in the relaxed (continuous) design parameter domain (1) is computed by a Feasible Sequential Quadratic Programming (FSQP) approach. ${ }^{11}$ The FSQP approach uses the performances at the worst case points to consider variations in operating and process conditions. To compute the worst case points efficiently, a successive approximation is realized. ${ }^{7}$

\subsection{FSQP for tolerance design}

For optimization problem (14), the Lagrangian function can be given as

$$
\mathcal{L}(\mathbf{d}, \boldsymbol{\lambda})=\varphi(\mathbf{d})-\sum_{i} \lambda_{i} c_{i}(\mathbf{d})
$$

In (18) $\varphi(\mathbf{d})$ is the objective function (13), $\lambda_{i}$ is the Lagrangian multiplier for constraint $c_{i}(\mathbf{d})$. Due to the Karush Kuhn Tucker conditions, the gradient of the Lagrangian function with respect to $\mathbf{d}$ and to $\boldsymbol{\lambda}$ must be zero at the optimum $\mathbf{d}^{*}$. This condition is used by Sequential Quadratic Programming (SQP) algorithms which compute a sequence of quadratic models of the Lagrangian function and 
improve the intermediate solutions iteratively using the optima of these quadratic models. For this purpose, the equation system

$$
\left(\begin{array}{cc}
\mathbf{H}^{(\mu)} & -\mathbf{J}^{(\mu)^{T}} \\
-\mathbf{J}^{(\mu)} & \mathbf{0}
\end{array}\right)\left(\begin{array}{c}
\Delta \mathbf{d}^{(\mu)} \\
\Delta \boldsymbol{\lambda}(\mu+1)
\end{array}\right)=\left(\begin{array}{c}
-\mathbf{g}^{(\mu)} \\
\mathbf{c}^{(\mu)}
\end{array}\right)
$$

is solved in each iteration $\mu$ of an SQP approach. $\mathbf{H}^{(\mu)}=\left.\nabla_{\mathbf{d d}} \mathcal{L}(\mathbf{d}, \boldsymbol{\lambda})\right|_{\mathbf{d}^{(\mu)} \boldsymbol{\lambda}^{(\mu)}}$ in (19) is the Hessian matrix. $\mathbf{J}^{(\mu)}=\left.\nabla_{\mathbf{d}} \mathbf{c}(\mathbf{d})\right|_{\mathbf{d}^{(\mu)}}$ and $\mathbf{c}^{(\mu)}$ is the Jacobian matrix and the vector of constraint values for active constraints, i.e., for constraints which prevent the objective function from further improve, at $\mathbf{d}^{(\mu)} \cdot \mathbf{g}^{(\mu)}=\left.\nabla_{\mathbf{d}} \varphi(\mathbf{d})\right|_{\mathbf{d}^{(\mu)}}-\mathbf{J}^{(\mu)} \boldsymbol{\lambda}^{(\mu)}$ is the derivative of (18) with respect to d. $\Delta \mathbf{d}^{(\mu)}$ and $\Delta \boldsymbol{\lambda}(\mu)$ is the change of design parameters and Lagrangian multipliers. The Hessian matrix $\mathbf{H}^{(\mu)}$ is iteratively approximated in this work by the BFGS (Broyden-Fletcher-Goldfarb-Shanno) approximation formula. Many SQP algorithms use line search approaches to compute a intermediate solution in direction of $\Delta \mathbf{d}^{(\mu)}$.

In contrast, the FSQP approach ${ }^{11}$ computes a descent direction and a correction step for this direction considering the nonlinearity of the constraints. Therefore, the approach can guarantee that the constraints are fulfilled in each iteration. The constraint fulfillment in each step is, however, crucial for deterministic analog sizing approaches to avoid the search in strongly nonlinear regions of the design space which can be typically found where design rules are violated.

To enlarge the FSQP approach for the consideration of variations in operating and process conditions, the objective function must be computed and the equation system (19) must be built up at the worst case points which depend on the currently selected design parameter point. An accurate computation of the worst case points during the analog sizing approach is computationally too costly as it would come along with a high number of required circuit simulations. Therefore, a successive approximation of the worst case points ${ }^{7}$ is used and the performances as well as the sensitivities of the performances — which are required to update (19) — are computed at these approximated worst case points.

To calculate performances and sensitivities at the worst case points, the algorithm in Fig. 2 is used. Initially (lines $1-6$ ) the sensitivity of the performances against variations in operating parameters is computed such that a linear model

$$
f_{i}\left(\mathbf{d}^{(\mu)}, \mathbf{o}, \mathbf{s}_{w, i}^{(\mu)}\right) \approx f_{i}\left(\mathbf{d}^{(\mu)}, \mathbf{o}_{w, i}^{(\mu)}, \mathbf{s}_{w, i}^{(\mu)}\right)+\mathbf{g}_{o, i}^{(\mu) T}\left(\mathbf{o}-\mathbf{o}_{w, i}^{(\mu)}\right)
$$

of each performance with respect to the operating parameters can be derived, with

$$
\mathbf{g}_{o, i}^{(\mu)}=\left.\nabla_{\mathbf{o}} f_{i}(\mathbf{d}, \mathbf{o}, \mathbf{s})\right|_{\mathbf{d}=\mathbf{d}^{(\mu)}, \mathbf{o}=\mathbf{o}_{w, i}^{(\mu)}, \mathbf{s}=\mathbf{s}_{w, i}^{(\mu)}} .
$$

Assuming an upper bound $f_{U, i}$ for each performance, the worst case points with respect to operating parameters $\mathbf{o}_{w, i}^{(\mu+1)}$ can be approximated by the maximum of the 


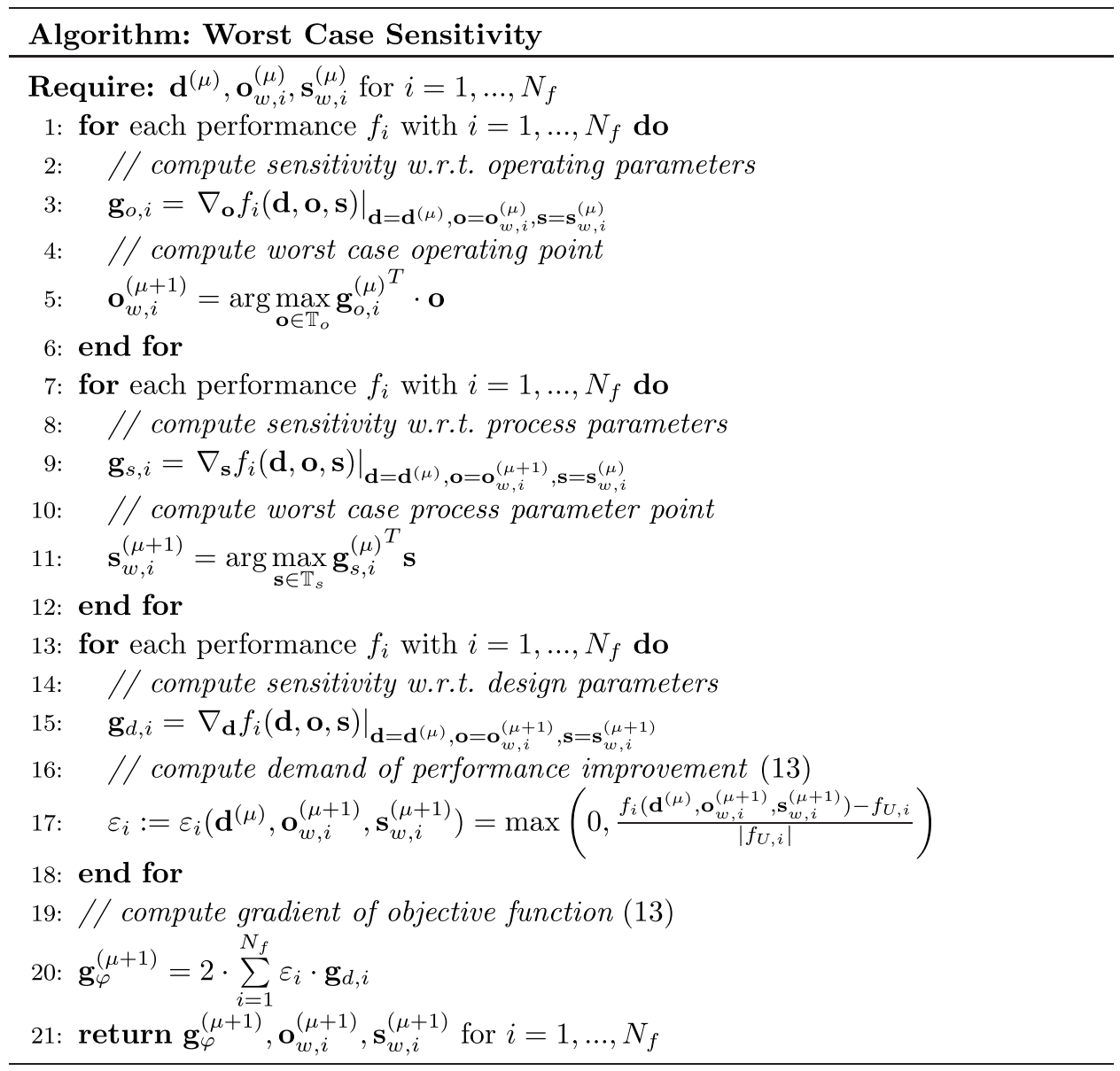

Fig. 2. Computation of the worst-case points and worst case sensitivities.

linear model in the operating region $\mathbb{T}_{o}(4),(16)$ :

$$
\mathbf{o}_{w, i}^{(\mu+1)}=\arg \max _{\mathbf{o} \in \mathbb{T}_{o}} \mathbf{g}_{o, i}^{(\mu) T} \mathbf{o} .
$$

Starting from the nominal value for operating and process parameters in the first iteration - i.e., with a classical worst case analysis ${ }^{6}$ - the model is successively refined at the newest available approximation of the worst case points. Accordingly, the worst case points for the process parameters are computed in lines 7-12 where the linear model of each performance is computed with respect to the process parameters. The worst case points of the performances in the tolerance ellipsoid $\mathbb{T}_{s}$ 
(8) are approximated on this linear model, i.e., by a realistic worst case analysis ${ }^{6}$ :

$$
\mathbf{s}_{w, i}^{(\mu+1)}=\arg \max _{\mathbf{s} \in \mathbb{T}_{s}} \mathbf{g}_{s, i}^{(\mu) T} \mathbf{s} .
$$

The sensitivity of the performances with respect to the process parameters

$$
\mathbf{g}_{s, i}^{(\mu)}=\left.\nabla_{\mathbf{s}} f_{i}(\mathbf{d}, \mathbf{o}, \mathbf{s})\right|_{\mathbf{d}=\mathbf{d}^{(\mu)}, \mathbf{o}=\mathbf{o}_{w, i}^{(\mu+1)}, \mathbf{s}=\mathbf{s}_{w, i}^{(\mu)}}
$$

is computed at the current design parameter point $\mathbf{d}^{(\mu)}$ and at the newest available approximations for the worst case points $\mathbf{o}=\mathbf{o}_{w, i}^{(\mu+1)}$ and $\mathbf{s}=\mathbf{s}_{w, i}^{(\mu)}$. The described procedure to compute the worst case points increases the accuracy of the approximation over the iterations. Although the result of the approach might not be the global worst case points, the sufficient accuracy of the method has been shown in previous publications. ${ }^{7,13}$

Besides the worst case points, the gradient of the objective function must be computed for the FSQP approach. For this purpose, the sensitivities against variations in the design parameters and the performance values are computed at the approximated worst case points in lines $13-18$. The results are used in line 20 to compute the gradient of the objective function at the worst case points, given for (13) by

$$
\begin{aligned}
\left.\nabla_{\mathbf{d}} \varphi(\mathbf{d})\right|_{\mathbf{d}^{(\mu)}} & \left.\approx \sum_{i=1}^{N_{f}} \nabla_{\mathbf{d}_{i}} \varepsilon_{i}\left(\mathbf{d}, \mathbf{o}_{w, i}^{(\mu+1)}, \mathbf{s}_{w, i}^{(\mu+1)}\right)^{2}\right|_{\mathbf{d}^{(\mu)}} \\
& =\left.\sum_{i=1}^{N_{f}} 2 \cdot \varepsilon_{i}\left(\mathbf{d}^{(\mu)}, \mathbf{o}_{w, i}^{(\mu+1)}, \mathbf{s}_{w, i}^{(\mu+1)}\right) \cdot \nabla_{\mathbf{d}_{i}}\left(\mathbf{d}, \mathbf{o}_{w, i}^{(\mu+1)}, \mathbf{s}_{w, i}^{(\mu+1)}\right)\right|_{\mathbf{d}^{(\mu)}}
\end{aligned}
$$

Using this gradient and the described approximation of the worst case points in the FSQP algorithm allows the computation of a tolerance design in the continuous domain. The realization of the algorithm using three subsequent for-loops allows a high parallelization of the simulations which are required to compute the performance sensitivities, and which have a strong influence on the algorithm runtime.

\subsection{Analog branch-and-bound approach}

The FSQP approach in Sec. 3.1 allows the computation of a tolerance design in a continuous domain. However, this work focuses on sizing problems with discrete parameters. To compute such a discrete solution, a new Branch-and-Bound approach is used.

It is assumed in this work that a solution for optimization problem (14) exists which fulfills (15). Therefore, a result is only of interest if it solves the sizing task. If these assumptions are not valid for the design problem, minor changes of the algorithm are required which would, however, come along with an increased runtime. 


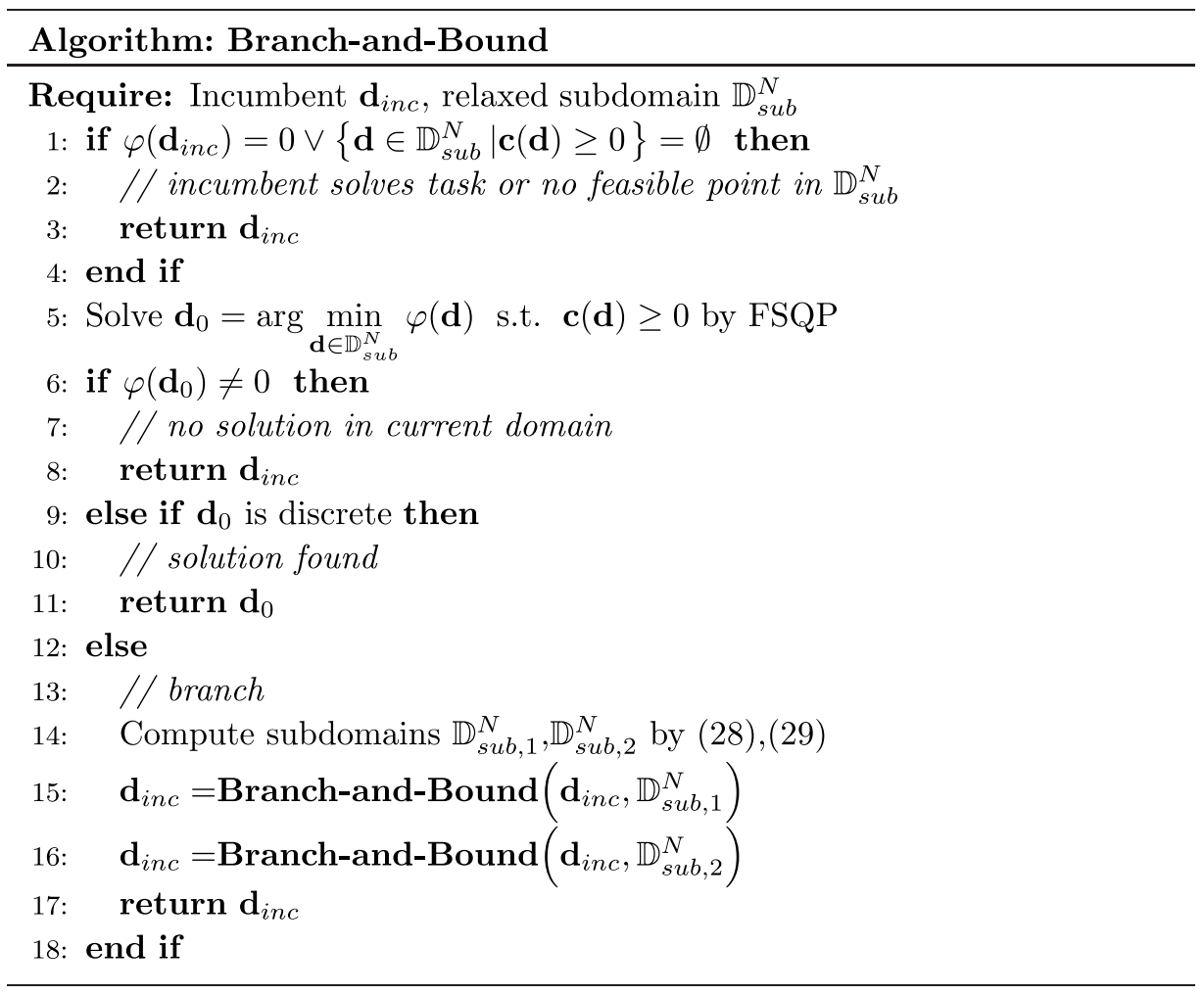

Fig. 3. Branch-and-Bound algorithm. ${ }^{14}$

The new Branch-and-Bound approach in this work is implemented as a recursive algorithm as shown in Fig. 3. In each recursion of the algorithm, a solution for the tolerance design is computed in a relaxed (continuous) subdomain of the relaxed design parameter domain $\mathbb{D}_{\text {rel }}^{N}$ (1) by the FSQP approach in Sec. 3.1 (line 5). A discrete solution for the sizing task can be found in a subdomain if and only if a continuous solution for the sizing task exists in this domain. Thus, a subdomain which does not include a solution for the sizing task in the continuous domain has not to be considered and can be pruned from $\mathbb{D}_{\text {rel }}^{N}$ (line 1 and 6). Otherwise, i.e., if the solution of the sizing task in the continuous domain solves the tolerance design problem, two cases can be found: If the solution of the continuous domain $\mathbf{d}_{0}$ is an element of the discrete domain $\mathbf{d}_{0} \in \mathbb{D}_{\text {disc }}^{N}$, a solution for the discrete tolerance design has been found. Thus, the approach can return the result as a discrete solution (line 9 ), store it as incumbent $\mathbf{d}_{\text {inc }}$ (line 15 or 16) to make it available for other recursions, and avoid the search in further subdomains (line 1). If the result in the continuous domain is, however, not in the discrete set $\mathbb{D}_{\text {disc }}^{N}$, the current relaxed subdomain is subdivided further. The new subdomains must be generated such that 
they are non-overlapping and such that the solution of all subdomains is discrete after a limited number of recursions.

To subdivide the search space as required, rounding constraints are used. I.e., considering the (continuous) solution $\mathbf{d}_{0}$ in the current subdomain, one component $d_{i}$ with value $d_{i, 0}$ and which must be discretized is take from $\mathbf{d}_{0}$. For this parameter two constraints

$$
\begin{aligned}
c_{\mathrm{up}}(\mathbf{d}) & :=d_{i}-\left\lceil d_{i, 0}\right\rceil \geq 0, \\
c_{\text {down }}(\mathbf{d}) & :=\left\lfloor d_{i, 0}\right\rfloor-d_{i} \geq 0
\end{aligned}
$$

are generated. The rounding operators $\lceil\bullet\rceil$ and $\lfloor\bullet\rfloor$ round the parameter to the next higher and lower discrete value according to (2). With these two rounding constraints, two new relaxed subdomains

$$
\begin{aligned}
& \mathbb{D}_{\mathrm{sub}, 1}^{N}=\left\{\mathbf{d} \in \mathbb{D}_{\mathrm{sub}}^{N} \mid c_{\mathrm{up}}(\mathbf{d}) \geq 0\right\} \\
& \mathbb{D}_{\mathrm{sub}, 2}^{N}=\left\{\mathbf{d} \in \mathbb{D}_{\mathrm{sub}}^{N} \mid c_{\text {down }}(\mathbf{d}) \geq 0\right\}
\end{aligned}
$$

are derived from the current subdomain $\mathbb{D}_{\text {sub }}^{N}$ in line 14 of the Branch-and-Bound algorithm. The domain between the constraints, i.e., $\left\lfloor d_{i, 0}\right\rfloor<d_{i}<\left\lceil d_{i, 0}\right\rceil$, does not contain any discrete point and is cut from the search space for the remainder of the algorithm.

The decision which parameter should be selected for branching — i.e., for which parameter the rounding constraints are constructed - is crucial for the efficiency of the algorithm and must be carefully adapted to the problem to be solved. The branching method in the new algorithm prefers the direction to the optimum of the sizing problem. I.e., given a quadratic model of the Lagrangian function and a nonzero gradient of the objective function at a point where the specifications are fulfilled at the worst case points - such a gradient is typically available if the computation of the demand of performance improvement is modified such that it becomes zero only if the specifications are slightly over-fulfilled - a direction of improvement $\Delta \mathbf{d}$ can be computed by solving (19). From the design parameters which must be discretized, the component index which corresponds to the largest absolute value in $\Delta \mathbf{d}$ is taken:

$$
i=\arg \max _{j}\left|\Delta d_{j}\right| \quad \text { s.t. } d_{j} \text { must be discretized } .
$$

The branching constraints are then constructed according to (26) and (27). From the two resulting subdomains, the subdomain which lies in the direction of $\Delta d_{i}$ is selected and considered at first in the Branch-and-Bound algorithm.

The Branch-and-Bound algorithm presented so far solves the analog sizing task under consideration of variations in operating and process conditions. However, the number of considered subdomains can be high. As each of the considered subproblems corresponds to a continuous tolerance design task and is solved by the simulation based FSQP approach, the runtime of the Branch-and-Bound algorithm 
presented so far can be high. Thus, besides the consideration of the rounded solution - which does not solve the sizing task in general but gains good results in certain cases - the expected discrete solution for the discrete sizing problem is predicted at the end of each FSQP run. ${ }^{2}$ For this purpose, the equation system in (19), which is computed during the FSQP approach, is interpreted as the quadratic programming problem

$$
\min \frac{1}{2} \Delta \mathbf{d}^{T} \mathbf{H} \Delta \mathbf{d}+\mathbf{g}^{T} \Delta \mathbf{d} \quad \text { s.t. } \mathbf{J} \Delta \mathbf{d}+\mathbf{c} \geq 0 .
$$

The discrete optimum of (31) in the current discrete subdomain $\mathbb{D}_{\text {disc }}^{N} \cap \mathbb{D}_{\text {sub }}^{N}$ solves the analog sizing task for a tolerance design in many cases without additional simulations such that the simulation based FSQP approach must be used only once. It can be noted that the computation of the optimum of (31) is not necessary if additional linear constraints are added which force that the performances are fulfilled in linear approximation, as in this case each point inside the linearly constrained region potentially solves the sizing task.

\section{Simulation Results}

The efficacy and efficiency of the algorithm can be verified by the three experiments presented in this section which reflect the typical performance of the new analog Branch-and-Bound algorithm (ABAB). The examples show the sizing of the Miller amplifier (MA) in Fig. 4, of the low noise amplifier (LNA) in Fig. 5, and of the low voltage amplifier (LVA) in Fig. 6 considering variations in operating and process conditions. The sizing is computed by the analog Branch-and-Bound algorithm in Sec. 3.2 and by a state-of-the-art approach. The new ABAB approach discretizes the result by recursively adding rounding constraints (26), (27) and by solving the quadratic subproblem (31). The used state-of-the-art approach (ROUND) computes a continuous solution and discretizes all discrete parameters at once by rounding the result to the next discrete point. As a consequence, the result of the state-of-the-art approach $^{10}$ is a discrete neighbor of the continuous solution ${ }^{8}$ while the result of $\mathrm{ABAB}$ might be any point in the discrete design space. For the sizing of all circuits, a

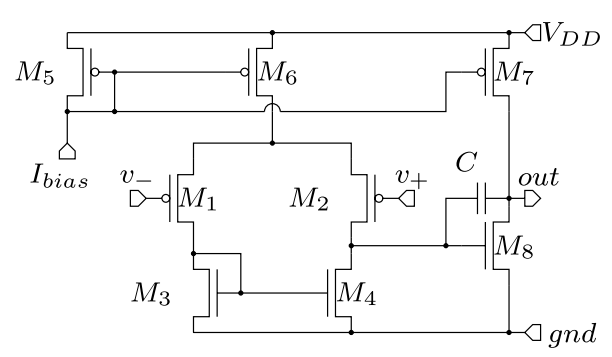

Fig. 4. Miller amplifier. 


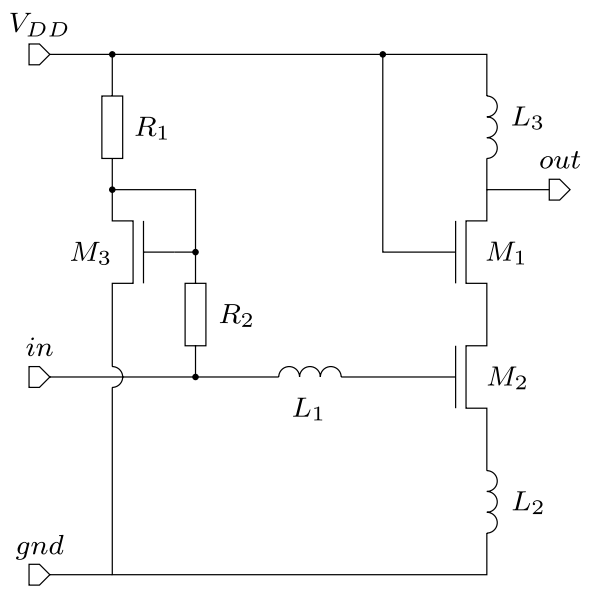

Fig. 5. Low noise amplifier. ${ }^{15}$

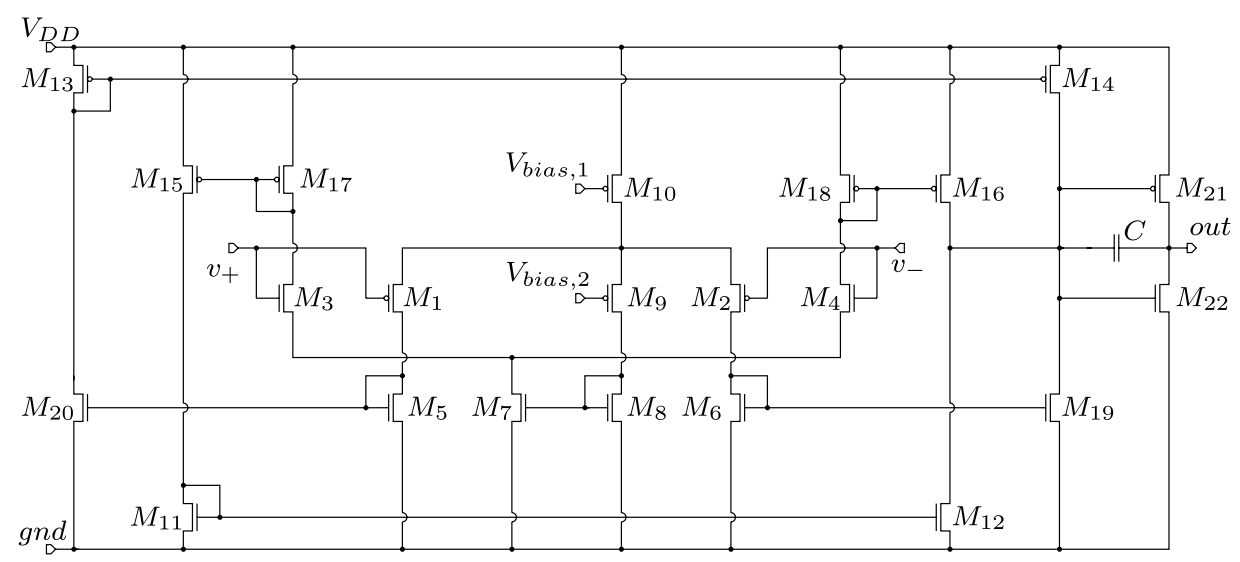

Fig. 6. Low voltage amplifier. ${ }^{16}$

$180 \mathrm{~nm}$ CMOS technology was used. All transistor lengths and widths were allowed to be scaled in the range from $300 \mathrm{~nm}$ to $1 \mu \mathrm{m}$ and from $300 \mathrm{~nm}$ to $10 \mu \mathrm{m}$ with a $10 \mathrm{~nm}$ manufacturing grid each. In addition the multipliers of the transistors were considered in the range from 1 to 100. Voltage sources, capacitors, and resistors were assumed to be ideal devices with continuous values between $0 \mathrm{~V}$ and $V_{D D}$, between $100 \mathrm{fF}$ and $100 \mathrm{pF}$, and between $100 \Omega$ and $10 \mathrm{k} \Omega$. For the sizing of the inductors in the LNA design a realistic inductor model ${ }^{17}$ was used which allows the sizing of the inductor using the inner diameter of the inductor, the width of the turns, the spacing between the turns, and the number of winding turns. The inner diameter in this experiment was scaled in the range from $20 \mu \mathrm{m}$ to $200 \mu \mathrm{m}$, the line width and the line spacing in 
the range from $2 \mu \mathrm{m}$ to $50 \mu \mathrm{m}$ and $1 \mu \mathrm{m}$ to $5 \mu \mathrm{m}$, each with a grid of $0.25 \mu \mathrm{m}$. The number of turns was assumed to be scalable in halve turns from 1.5 to 20 .

For the MA, the lengths of all NMOS and of all PMOS transistors were matched as well as all transistor lengths in the sizing of the LVA and of the LNA. In addition, the multipliers and widths of all transistors in the same differential pair, in the same current mirror and in the same current mirror bank were set equal and the symmetries of the circuits were considered to match further parameters as, e.g., the multipliers and widths of the transistors $M_{17}$ and $M_{18}$ of the LVA. This setup allows a layout friendly sizing in the sense that no rounding operations are required for the layout if the transistor gates should lie on a manufacturing grid or if certain structures should be realized as multifinger or common centroid structures. After setting the required transistor parameters to be equal, 12 discrete parameters and one continuous parameter (the capacitance) were considered for the sizing of the MA. For the sizing of the LVA the bias voltages and the compensation capacitance were considered as continuous parameters such that 26 discrete and three continuous design parameters can be found for this task. The sizing of the LNA requires the consideration of the two continuously scalable resistances as well as of 19 discrete design parameters for the inductors and transistors.

For the tolerance design three, in case of the MA four, different operating conditions were defined for each circuit as shown in Table 1. In addition, normal distributed global variations of electron mobility and threshold voltages for NMOS and PMOS transistors, and of the oxide thickness were considered as process variations, as well as normal distributed local variations of electron mobility and threshold voltages for each single transistor in case of MA and LNA. Thus, a total of 21 process parameters for the MA, 5 for the LVA, and 9 for the LNA was used. A minimum yield of $99.9 \%\left(\beta_{w}=3\right.$ in (8)) was required per performance. As for MA and LVA the eight performances in Table 2 and for the LNA the six performances in Table 3 were

Table 1. Operating parameters for Miller (MA), low voltage (LVA), and low noise amplifier (LNA).

\begin{tabular}{lccc}
\hline & Min & Nominal & Max \\
\hline $\mathrm{MA}$ & & & \\
$V_{D D}(\mathrm{~V})$ & 2.3 & 2.5 & 2.7 \\
$C_{\text {load }}(\mathrm{pF})$ & 15 & 20 & 25 \\
$T\left({ }^{\circ} \mathrm{C}\right)$ & -40 & 27 & 125 \\
$I_{\text {bias }}(\mu \mathrm{A})$ & 9 & 10 & 11 \\
$\mathrm{LVA}$ & & & \\
$V_{D D}(\mathrm{~V})$ & 1.9 & 2.0 & 2.1 \\
$C_{\text {load }}(\mathrm{pF})$ & 1.9 & 2 & 2.1 \\
$T\left({ }^{\circ} \mathrm{C}\right)$ & -40 & 27 & 125 \\
$\mathrm{LNA}$ & & & \\
$V_{D D}(\mathrm{~V})$ & 2.4 & 2.5 & 2.6 \\
$C_{\text {load }}(\mathrm{fF})$ & 90 & 100 & 110 \\
$T\left({ }^{\circ} \mathrm{C}\right)$ & -40 & 27 & 85 \\
\hline
\end{tabular}


Table 2. Specified gain, PSRR, CMRR, phase margin (PM), transit frequency $\left(f_{t}\right)$, rising and falling slew-rate $(\mathrm{SR}+, \mathrm{SR}-)$, and Power for Miller (MA) and low voltage amplifier (LVA).

\begin{tabular}{lcccccccc}
\hline & $\begin{array}{c}\text { Gain } \\
(\mathrm{dB})\end{array}$ & $\begin{array}{c}\text { PSRR } \\
(\mathrm{dB})\end{array}$ & $\begin{array}{c}\mathrm{CMRR} \\
(\mathrm{dB})\end{array}$ & $\begin{array}{c}\mathrm{PM} \\
\left({ }^{\circ}\right)\end{array}$ & $\begin{array}{c}f_{T} \\
(\mathrm{MHz})\end{array}$ & $\begin{array}{c}\mathrm{SR}+ \\
(\mathrm{V} / \mu \mathrm{s})\end{array}$ & $\begin{array}{c}\mathrm{SR}- \\
(\mathrm{V} / \mu \mathrm{s})\end{array}$ & $\begin{array}{c}\text { Power } \\
(\mathrm{mW})\end{array}$ \\
\hline MA & $>75$ & $>140$ & $>120$ & $>60$ & 10 & $>8$ & $<-8$ & $<5$ \\
LVA & $>75$ & $>90$ & $>90$ & $>60$ & 20 & $>12$ & $<-12$ & $<7$ \\
\hline
\end{tabular}

Table 3. Specified s-parameters, third-order input intercept point, and noise factor of the LNA for a center frequency of $2.4 \mathrm{GHz}$.

\begin{tabular}{lccccc}
\hline$S_{11}(\mathrm{~dB})$ & $S_{12}(\mathrm{~dB})$ & $S_{21}(\mathrm{~dB})$ & $S_{22}(\mathrm{~dB})$ & $\mathrm{IIP} 3(\mathrm{dBm})$ & $\mathrm{NF}(\mathrm{dB})$ \\
\hline$<-9$ & $<-30$ & $>15$ & $<-5$ & $>-5$ & $<2.5$ \\
\hline
\end{tabular}

specified, the minimum required parametric yield at the worst case operating points is defined as $99.9 \%^{8} \approx 99.0 \%$ for MA and LVA and $99.9 \%^{6} \approx 99.2 \%$ for the LNA. Typical analog design rules ${ }^{12}$ were considered for all sizing tasks.

The yield for each result computed by the new approach was evaluated by a Monte Carlo analysis with 2500 samples at the worst case operating points and is listed in Table 4 . It can be clearly seen that - starting from a nominal point where the yield requirements were not fulfilled - the new approach can always find a solution. In contrast, the state-of-the-art approach missed the specified yield requirements in all examples and cannot be used to compute a reliable result in practice. Especially the clearly improved yield for the LNA is notable. In this case, the rounding operation in ROUND causes a deterioration of the input matching of the circuit while good matching can be observed for the result of ABAB. As a consequence, the yield at the solution computed by ABAB is much higher.

The efficiency of the new analog Branch-and-Bound approach can be seen if the runtime of this new approach is compared to the state-of-the-art approach. The

Table 4. Yield at worst-case operating points for nominal design (NOM), continuous sizing and rounding (ROUND), and sizing with the new approach (ABAB) for Miller (MA), low voltage (LVA), and low noise amplifier (LNA) approximated by Monte Carlo analyses with 2500 samples at the worst case operating points.

\begin{tabular}{lccc}
\hline & NOM $(\%)$ & ROUND $(\%)$ & ABAB $(\%)$ \\
\hline MA & 0 & 86.0 & 99.8 \\
LVA & 0 & 98.0 & 99.4 \\
LNA & 0 & 14.6 & 99.9 \\
\hline
\end{tabular}


measured time in the examples was with $49 \mathrm{~min}$ for the MA, $120 \mathrm{~min}$ for the LVA, and $46 \mathrm{~min}$ for the LNA only 5, 7 and 6 min higher than the runtime of continuous sizing with subsequent rounding if $\mathrm{ABAB}$ and ROUND were 16 times parallelized and if the experiments were run on an 8 Core Intel R Xeon R X5500 2.67 GHz CPU with hyper threading. This additional runtime can be neglected if the benefit of the increased yield is considered.

The runtime of $\mathrm{ABAB}$ is approximately the product of number of sequential simulations and time per simulation. The algorithm is optimized with respect simulation number and the runtime can be further reduced by higher parallelization if more CPUs and simulator licenses are available. Therefore, ABAB is also suitable in sizing analog blocks with high parameter and device numbers, e.g., ADCs, if the simulation time for the block is low. A low simulation time can be achieved, e.g., by providing efficient simulation models. Alternatively, large circuits can be partitioned into sub-blocks which can be sized independently. ${ }^{18}$

\section{Conclusion}

A new approach for the sizing of analog circuits under consideration of variations in operating and process conditions and discrete parameters was presented in this paper. The new method solves the problem using Branch-and-Bound, FSQP, and a successive linear approximation of the worst case points for operating and process conditions. Simulation results show that the highly efficient new algorithm can compute a sizing which is robust against variations, and that the runtime of the algorithms is negligibly higher than the runtime of a fast state-of-the-art approach which cannot reliably solve the problem.

\section{References}

1. E. J. Nowak, I. Aller, T. Ludwig, K. Kim, R. V. Joshi, C.-T. Chuang, K. Bernstein and R. Puri, Turning silicon on its edge [double gate CMOS/FinFET technology], IEEE Circuits Devices Mag. 20, August 2004.

2. M. Pehl and H. Graeb, An SQP and Branch-and-Bound Based Approach for Discrete Sizing of Analog Circuits, InTech, February 2011, pp. 297-316.

3. M. Pehl and H. Graeb, RaGAzi: A random and gradient-based approach to analog sizing for mixed discrete and continuous parameters, IEEE ISIC (2009).

4. R. Phelps, M. Krasnicki, R. A. Rutenbar, L. R. Carley and J. R. Hellums, Anaconda: Simulation-based synthesis of analog circuits via stochastic pattern search, IEEE TCAD 19, June 2000.

5. A. Somani, P. P. Chakrabarti and A. Patra, An evolutionary algorithm-based approach to automated design of analog and RF circuits using adaptive normalized cost functions, IEEE TEC 11, June 2007.

6. H. Graeb, Analog Design Centering and Sizing (Springer, 2007).

7. R. Schwencker, F. Schenkel, M. Pronath and H. Graeb, Analog circuit sizing using adaptive worst-case parameter sets, DATE (2002). 
8. D. Li and X. Sun, Nonlinear Integer Programming (Springer, New York, USA, 2006).

9. F. Gong, X. Liu, H. Yu, S. X. D. Tan, J. Ren and L. He, A fast non-Monte Carlo yield analysis and optimization by stochastic orthogonal polynomial, ACM TODEAS 17, January 2012.

10. WiCkeD, www.muneda.com (2011).

11. C. Lawrence and A. Tits, A computationally efficient feasible quadratic programming algorithm, SIOPT 11 (2000).

12. T. Massier, H. Graeb and U. Schlichtmann, The sizing rules method for CMOS and bipolar analog integrated circuit synthesis, IEEE TCAD 27 (2008) 2209-2222.

13. D. Mueller-Gritschneder and H. Graeb, Computation of yield-optimized pareto fronts for analog integrated circuit specifications, DATE, (2010).

14. M. Pehl, M. Zwerger and H. Graeb, Variability-aware automated sizing of analog circuits considering discrete design parameters, IEEE ISIC (2011).

15. T. H. Lee, The Design of CMOS Radio-Frequency Integrated Circuits (Cambridge University Press, Cambridge, UK, (2004).

16. R. Martins, On the Design of Very Low Power Integrated Circuits, $\mathrm{PhD}$ thesis, Vienna University of Technology (1998).

17. P. Pereira, M. H. Fino and F. V. Coito, Using discrete-variable optimization for cmos spiral inductor design, ICM (2009).

18. J. Zou, D. Mueller and H. Graeb, A CPPLL hierarchical optimization methodology considering jitter, power and locking time, IEEE DAC (2006). 